\title{
A genome-wide analysis of the cellulose synthase-like (Csl) gene family in maize
}

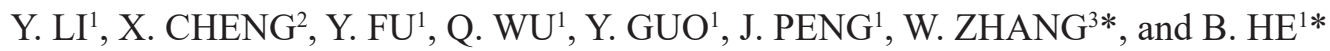 \\ College of Life Sciences, Jiangxi Science and Technology Normal University, Nanchang 330013, P.R. China ${ }^{l}$ \\ College of Life Sciences, Sichuan University, Chengdu 610064, P.R. China ${ }^{2}$ \\ College of Life Sciences and Technology, Honghe University, Mengzi 661100, P.R. China ${ }^{3}$
}

\begin{abstract}
Cell walls play an important role in the structure and morphology of plants as well as in responses to various biotic and abiotic stresses. Although the comprehensive analysis of genes involved in cellulose synthase has been performed in model plants, such as Arabidopsis thaliana and rice, information regarding cellulose synthase-like (Csl) genes in maize is limited. In this study, a total of 56 members of $C s l$ gene family were identified in maize genome and classified into six subfamilies. Analysis of gene structure and conserved motif indicated functional similarities among the ZmCsl proteins within the same subfamily. Additionally, the $56 \mathrm{ZmCsl}$ genes were dispersed on 10 chromosomes. The expression patterns of $\mathrm{ZmCsl}$ genes in different tissues using the transcriptome data revealed that most of $\mathrm{ZmCsl}$ genes had a relatively high expression in root and tassel tissues. Moreover, the expression profiles of $\mathrm{ZmCsl}$ genes under drought and re-watering indicated that the expression of $\mathrm{ZmCsl}$ genes were mainly responsive to early stage of drought stress. The protein-protein interaction network of $\mathrm{ZmCsl}$ proposed some potentially interacting proteins. The data presented a comprehensive survey of $C s l$ gene family in maize. The detailed description of maize $C s l$ genes will be beneficial to understand their structural, functional, and evolutionary features and provide an important foundation for studying the roles of $\mathrm{ZmCsl}$ genes in response to biotic and abiotic stresses.
\end{abstract}

Additional key words: drought, $\mathrm{ZmCls}$ gene structure and function, protein-protein interaction, rehydration, Zea mays.

\section{Introduction}

The plant cell walls are essential for defining the shape of cells as well as providing structural integrity to plant tissues and organs. As the primary barrier, plant cell wall serves essential functions in resisting high cellular pressure potential and in protection of plants against environmental impacts (Kaur et al. 2017). Plant cell wall mainly consists of multiple polysaccharide fractions (Kaur et al. 2016). Among the various classes of polysaccharides, the major load-bearing component is cellulose. Cellulose is synthesized by cellulose synthase like (Csl) proteins, which belong to the superfamily of glycosyl transferase 2 (GT2). Due to the importance of the plant cell wall, the synthesis of cellulose and Csl genes have become the focus of research interest.

The $C s l$ genes are found throughout the plant kingdom. However, a variable number of $\mathrm{Csl}$ genes ranging from
30 to 50 have been reported in different plant species. The members of $C s l$ gene family are classified into nine subfamilies: CslA, CslB, CslC, CslD, CslE, CslF, CslG, $C s l H$, and $C s l J$. Recently, a number of plant Csl genes have been identified, including rice (Hazen et al. 2002), poplar (Djerbi et al. 2005, Suzuki et al. 2006), and the moss Physcomitrella patens (Roberts and Bushoven 2007). The CslJ is a new Csl family discovered in cereals (Yin et al. 2009) whereas $C s l B$ and $C s l G$ families are generally lacked in cereals (Yin et al. 2014). Among the nine subfamilies, $C s l A, C s l C$, and $C s l D$ are conserved in all land plants whereas $C s l F$ and $C s l H$ families are restricted to grasses (Schreiber et al. 2014).

Several members of the $C s l$ subfamilies have been reported to be involved in the biosynthesis of different cell wall polysaccharides. For example, $C s l A$ and $C s l C$ genes, encoding 1,4- $\beta$-glucoside synthase, have been identified and they are involved in the biosynthesis of mannan

Submitted 20 March 2019, last revision 23 April 2019, accepted 19 June 2019.

Abbreviations: Csl - cellulose synthase-like; PPI - protein-protein interaction.

Acknowledgements: This study was funded by the project of young and middle-aged talent of Yunnan province (Grant No. 2017HB076), the teaching reform project of Jiangxi Science \& Technology Normal University (JGYB-17-78-16), the Science and Technology Research Project of Jiangxi Provincial Department of Education (GJJ180605), and the Doctor and master specific projects of Honghe University (XJ17B09). The first two authors contributed equally to this work.

* Corresponding authors; e-mails: zw_biology2@126.com; hebin.li@foxmail.com. 
and xylan, respectively (Cocuron et al. 2007, Liepman et al. 2005). Vega-Sánchez et al. (2012) have confirmed that rice CslF6 gene mediates MLG (mixed-linkage glucan) biosynthesis after over-expression in Nicotiana benthamiana, while Schreiber et al. (2014) reported that the $C s l F$ gene family in barley may be involved in $(1,3 ; 1,4)-\beta$-glucan synthesis. From all Csl families, CslD, encoding $1,4-\beta$-linked glucan synthase, has the oldest intron-exon structure and it is found in all sequenced terrestrial plant genomes, including Physcomitrella and Selaginella, indicating that it has a highly conserved function in the entire plant community (Verhertbruggen et al. 2011, Douchkov et al. 2016). However, CslD proteins have also been suggested to synthesize xylan, homogalacturonan, or mannan polysaccharides. Therefore, the true nature of $\mathrm{Csl}$ biochemical activity remains an open question.

Maize is one of the three major food crops with an ancient cultivation history, and has great potential for the development of high nutrition food as well as biofuel (Wright et al. 2005). The synthesis of cellulose is of vital importance to protect maize against environmental impacts and provide raw materials for industrial applications. However, there are few reports on the related genes and mechanisms of cellulose synthesis in maize. Therefore, the aim of this study was to identify the $C s l$ family members in the whole maize genome through large-scale data mining, to provide a basis for the cloning of $C s l$ gene family in maize, and to analyze its evolution and structure. Furthermore, the transcriptomic expression analysis of $\mathrm{ZmCsl}$ genes and the results of protein-protein interaction prediction can lay a good foundation for further study on the function and the mechanism of cellulose synthesis.

\section{Materials and methods}

Determination the $C s l$ family members in maize genome: Because naming rice $C s l$ genes is well documented, it is used as a reference. So, all the sequences of rice $C s l$ genes $(\mathrm{OsCs} l)$ were downloaded from the Rice Genome Annotation Project (http://rice.plantbiology. msu.edu/index.shtml). The whole genome sequence of maize was downloaded from NCBI. To identify the Csl gene family members in maize, the genome sequence of maize was aligned with all rice $C s l$ genes using the $N C B I$ $B L A S T-2.7 .1+$ software. The E-value was set to $1 \mathrm{e}^{-5}$. Then, the identity and cover region (more than $50 \%$ ) were used as filter criteria to eliminate improper Csl genes. These genes were further filtered through domain analysis to ensure that the selected sequences were nonredundant sequences to ultimately determine the candidate $C s l$ gene family members. All candidate members should contain one of the two Pfam domain models (PF00535 and PF03552).

Multiple sequence alignment and the construction of phylogenetic tree: Multiple sequence alignment of rice $\mathrm{Csl}$ genes and $\mathrm{ZmCsl}$ gene family members was performed by the Muscle program with the default parameters.
MEGA (v. 5.0.1.102) was applied to construct a maximum likelihood tree (Tamura et al. 2011). The phylogenetic tree was evaluated by the bootstrap method, and the repeat value was set to 1000 . The Figtree program (v. 1.4.3) was used to visualize it.

Analysis of conserved motif and gene structure: To identify the conserved motifs of each $\mathrm{ZmCsl}$ gene, deduced $\mathrm{ZmCsl}$ protein sequences were subjected to $M E M E$ v. 4.12.0. The parameters were set to zero or one of a contributing motif site per sequence, the number of motif was chosen to be five; the motif width was set 6 to $50(\mathrm{Wu}$ et al. 2016). The results are visualized by the TBtools software. To further study the characteristics of $\mathrm{ZmCsl}$ genes, we used the GSDS2.0 software to analyze the structure and distribution of introns and exons for each gene. The corresponding CDS sequences and genomic sequences were found in the $N C B I$ database according to the GenBank accession number. To determine the chromosomal locations of all $C s l$ genes in maize, the information of locus coordinates was obtained from $N C B I$. The location and relative distance of $C s l$ gene were displayed by the MapChart (v. 3.2) software (Voorrips et al. 2002).

Expression of $\mathrm{ZmCsl}$ during drought stress and in different tissues: The genome-wide transcriptome data of maize under drought were obtained from NCBI SRA databases from accession SRR6665368 to SRR6665379. Seeds of Zea mays L. inbred line B73 were used as experimental material and soaked with water at $28{ }^{\circ} \mathrm{C}$ for $36 \mathrm{~h}$. After germination, the seedlings were cultured under an irradiance of $600 \mu \mathrm{mol} \mathrm{m} \mathrm{m}^{-2} \mathrm{~s}^{-1}$, a 16-h photoperiod, day/ night temperatures of $24 / 18{ }^{\circ} \mathrm{C}$, and an air humidity of $60 \%$ for approximately $12 \mathrm{~d}$. When the seedlings had thre fully expanded leaves, $20 \%(\mathrm{~m} / \mathrm{v})$ polyethylene glycol (PEG) 6000 was added to the nutrient solution and after $60 \mathrm{~h}$ or $96 \mathrm{~h}$ of drought stress and after recovery for $3 \mathrm{~d}$ (denoted as T60, T96, and TR3d, respectively) leaves were harvested and immediately frozen in liquid nitrogen. Control seedlings were grown under the same conditions but without PEG treatment (denoted as CK60, CK96h, and CKR3d respectively).

The transcriptome data of different maize tissues have been submitted to NCBI SRA databases under accession No. SRP067440 (Wang et al. 2016). Roots were collected from 14-d-old seedlings; tassels and ears were collected during vegetative stages 7 and 8, pollen during the first reproductive stage, and embryo and endosperm from seeds $20 \mathrm{~d}$ after pollination. Tissues were immediately frozen in liquid nitrogen. The gene expressions were estimated by the TopHat/Cufflinks pipeline described in previous reports with fragments per kilobase of exon per million fragments mapped FPKM values. The heat maps for expression profiles were generated with the OmicShare tools (http:// www.omicshare.com/tools/Home/Index/index.html).

Construction of protein-protein interaction network: Protein-protein interaction (PPI) data were obtained from the online database of STRING, which is an open 
source software for predicting and visualizing complex networks. These interactions were derived from literature of experimental validation including physical interactions and enzymatic reactions found in signal transduction pathways. The PPI data were pre-processed including removing redundancy and self-loops (Zhou et al. 2016). Targets with a high confidence score $>0.7$ were selected to construct the PPI networks (Zhu et al. 2016). The PPI networks were visualized in Cytoscape with the nodes representing proteins/genes and the edges representing interactions between any two proteins/genes.

\section{Results}

A total of $59 \mathrm{Csl}$ genes were identified from the genome of maize using the $B L A S T$ programs with the query sequences of rice $C s l$ genes. Subsequently, database searches for maize, using conserved Pfam PF00535 and PF03552, resulted in the identification of $56 \mathrm{ZmCsl}$ genes. The identified genes were named following the nomenclature of rice, which shares synteny with maize (Table 1 Suppl.). The length of protein ranged from 136 to 1514 aminoacids. All ZmCsl analyzed in this study possessd the structures typical of the Csl family, including a glycosyl transferase domain (PF00535) or cellulose synthase domain (PF03552). Except the structures mentioned above, ZmCslD1, ZmCslD2-7, ZmCslD3, and ZmCslD4-3 had a RING/Ubox like zinc-binding domain (PF14570) whereas ZmCslD2-1, ZmCsiD2-3, ZmCsiD2-4, and ZmCslD2-6 had a zinc-binding RING-finger domain (PF14569).

To investigate the phylogenetic relationship of the $C s l$ gene family in maize and rice, the amino acid sequences of $56 \mathrm{Csl}$ proteins from maize and 34 from rice were

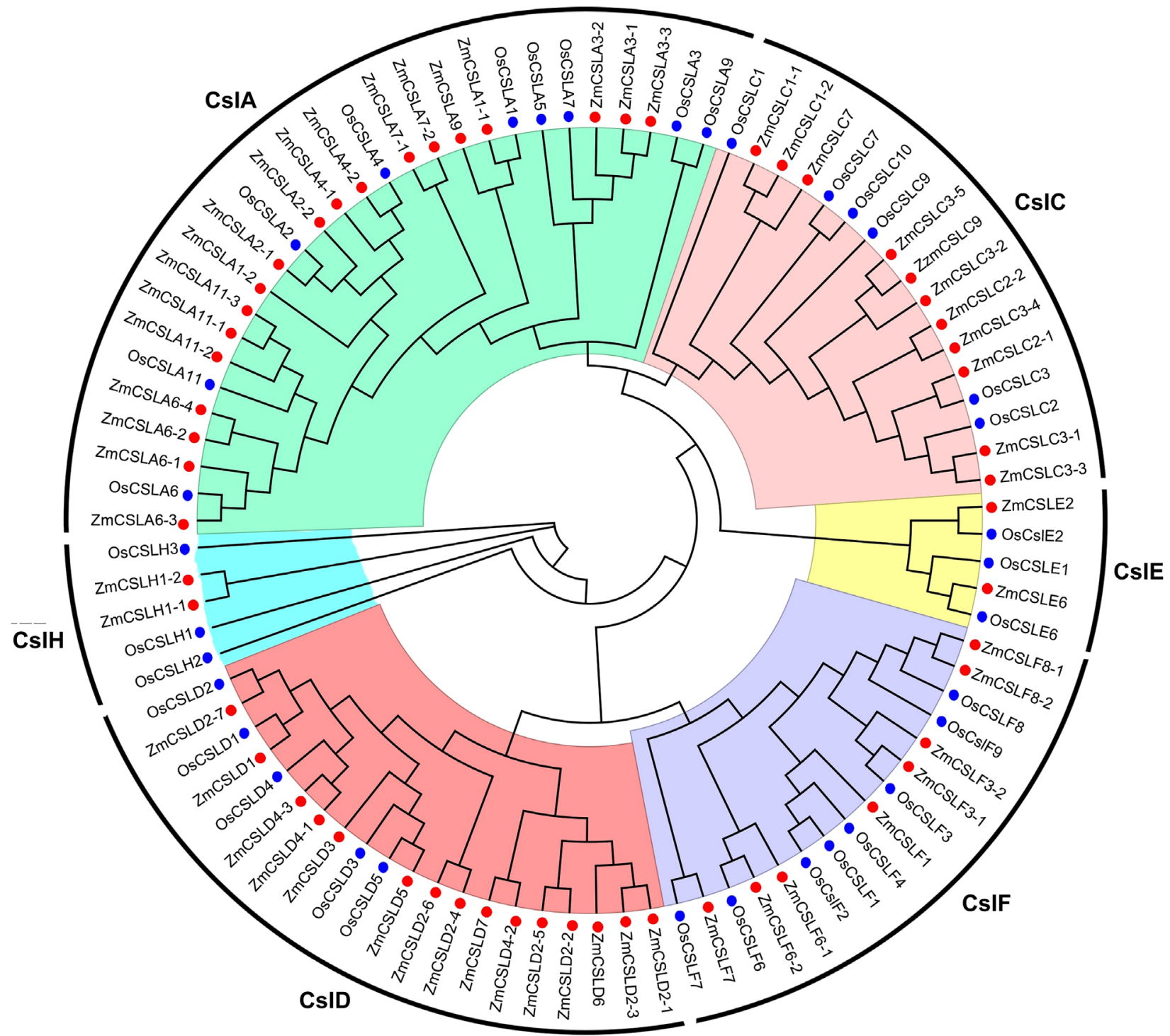

Fig. 1. A phylogenetic relationship of rice and maize cellulose synthase-like (Csl). The maximum likelihood tree was constructed by the $M E G A$ program with 1000 bootstrap sampling using full-length sequences of 56 maize and 34 rice Csl proteins. 
used to construct a phylogenetic tree. According it, maize Csl were classified into the same categories as rice Csl, which include CslA, CslC, CslD, CslE, CslF, and $\mathrm{CslH}$ subfamilies (Fig. 1). The CslB and CslG subfamilies, which lacked in cereals generally, missed also in maize. In addition, the CslJ subfamily, which was a new Csl family discovered in cereals, missed in maize as well because the sequences were unavailable. There were 19 members from maize and 9 from rice in the CslA subfamily, which was the largest subfamily. In addition, there were 11 and 13 members from maize classified into $\mathrm{CslC}$ and CslD subfamilies, only 2 members in $\mathrm{CslH}$ and $\mathrm{CslE}$ subfamilies, and $10 \mathrm{ZmCsl}$ grouped in the CslF subfamily. In rice, 6 and 5 OsCsl members were classified into CslC and CslD subfamilies whereas 10 OsCsl grouped in the CslF subfamily. In the six clades, $C s l$ genes had their clear orthologs in the genome of $O$. sativa, which suggests that these genes might be conserved for some specific functions in the two species. Furthermore, many $C s l$ genes were grouped together suggesting that these homologous genes may have derived from multiple duplications after the speciation of maize during the evolution.

Analysis of exon-intron structure in a gene family is beneficial to obtain a further insight into their evolutionary trajectory. We used the GSDS software to compare the fulllength cDNA with its corresponding genome sequence to detect the exon-intron structure of $Z m C s l$ genes. The results indicate that the exon-intron structure of $Z m C s 1$ genes was conserved to a certain degree within subfamilies, which supportS the evolutionary relationships among members of each clade. However, as shown in Fig. 2, the number of introns in $\mathrm{ZmCsl}$ genes was highly variable among different clades, ranging from 1 to 13 introns. For example, $\mathrm{ZmCslF}$ genes contained only 1 or 2 introns whereas the majority of $\mathrm{ZmCsl}$ genes in other subfamilies contained more introns. The large variation in structures of $\mathrm{ZmCsl}$ genes suggests that the maize genome has changed significantly during its long evolutionary history.

Toobtaininsights into the diversity ofmotifcompositions in $\mathrm{ZmCsl}$ proteins, putative motifs were predicted using the $M E M E$ program. A total of 10 conserved motifs were identified. The relative location of these motifs within the protein is represented in Fig. 3. The identified consensus sequence for the motifs is shown in Table 1. As expected, members that had similar motif compositions could be clustered into one class suggesting functional similarities among the $\mathrm{ZmCsl}$ proteins within the same subfamily. The $\mathrm{ZmCslA}$ and $\mathrm{ZmCslC}$ members, which have a closer phylogenetic relationship, contained motif 1 , motif 2 , motif 3 , motif 4 , motif 5 , motif 9 , and motif 10 whereas motif 6 , motif 7 , and motif 8 were found among other ZmCsl members. The distribution of motifs in different subfamilies implies sources of functional differentiation in $\mathrm{ZmCsl}$ genes in the evolutionary processes. Additionally, the motif distribution further confirms the accuracy of the phylogenetic relationship of $\mathrm{ZmCsl}$ genes.

The chromosomal distribution of the $\mathrm{ZmCsl}$ genes was visualized by the MapChart program (Fig. 4). The $56 \mathrm{ZmCsl}$ genes were dispersed on 10 chromosomes. Chromosomes 1 and 7 harbored the most (10 of 56) $\mathrm{ZmCsl}$ genes whereas $3 \mathrm{ZmCsl}$ genes were found on chromosome 8. In addition, there were $6 \mathrm{ZmCsl}$ genes on chromosomes 5 and 9.

To investigate the expression patterns of $\mathrm{ZmCsl}$ genes in different tissues, we utilized publicly available transcriptome data to survey the transcription of the $\mathrm{ZmCsl}$ genes in the ear, embryo, endosperm, pollen, root, and tassel. From the results, we observed that 20 out of $\mathrm{ZmCsl}$ genes had relatively high expressions in root tissue, mainly including $Z m C s l D$ and $Z m C s l F$ genes (Fig. 5). Moreover, there were $17 \mathrm{ZmCsl}$ genes that had very high transcript abundances in tassel, mainly $Z m C s l D, Z m C s l A$, and $\mathrm{ZmCslC}$ genes. Besides, six genes (ZmCslA3-1, ZmCslA3-2, ZmCslA3-3, ZmCslC1-1, ZmCslD4-1, and $\mathrm{ZmCslD4-3)}$ and two genes (ZmCslD5 and $\mathrm{ZmCslD9)}$ were highly expressed in ear and pollen, respectively.

In order to elucidate the roles of $\mathrm{ZmCsl}$ genes in response to drought stress induced by PEG addition, we analyzed their expression profiles under drought and recovery. Obviously, more $\mathrm{ZmCsl}$ genes were up-regulated after drought treatment for $60 \mathrm{~h}$ (Fig. 6). For example, ZmCslF6-1, ZmCslD1, ZmCslC2-1 and $\mathrm{ZmCslC2-2}$ displayed maximum expressions after stopping water supply for $60 \mathrm{~h}$. Some genes were lightly up-regulated such as $Z m C s l D 2-2$ and $Z m C s l D 4-2$. However, when recovering water supply, only $Z m C s l D 6, Z m C s l C 2-1$, and $\mathrm{ZmCs} l \mathrm{C2}-2$ exhibited high transcript accumulations. The

Table 1. The MEME motif sequences and lengths of maize cellulose synthase-like (ZmCsl) proteins.

\begin{tabular}{cllll}
\hline Motif & Conserved amino acid sequences & E-values & Sites & Width \\
\hline 1 & KGWKFIYVGDVKVKSELPSTYKAYRKQQHRWSCGPANLFRKMFPEIJKSK & $5.1 \mathrm{e}-953$ & 27 & 50 \\
2 & LNFFGFNGTAGVWRISAJEESGGWKDRTTVEDMDJAVRAHL & $3.0 \mathrm{e}-965$ & 30 & 41 \\
3 & IFDADFQPEPDFLKRTVPFLVHNPEJALVQARWSFVNKDENLLTRJQEMN & $3.8 \mathrm{e}-1044$ & 29 & 50 \\
4 & SGYYPMVLVQIPMYNEREVYKLSIGAACGLDWPRDRFLVQVLDDSTDPVI & $3.8 \mathrm{e}-868$ & 25 & 50 \\
5 & SFHFVIFWILFENVMSVHRFKAAVSGLLZLG & $4.2 \mathrm{e}-475$ & 27 & 31 \\
6 & AYVQFPQRFDGIDPTDRYANHNRVFFDGNMRGLDGJQGPVYVGTGCVFRR & $1.6 \mathrm{e}-861$ & 26 & 50 \\
7 & PMLVYVSREKRPGYDHHKKAGAMNALVRVSAVLSNAPFILNLDCDHYVNN & $7.2 \mathrm{e}-824$ & 23 & 50 \\
8 & IYGSVTEDVVTGFRMHNRGWRSVYCSPKRDAFRGTAPINLTDRLHQVLRW & $1.8 \mathrm{e}-794$ & 24 & 50 \\
9 & KWAQKGVNIKYEHRVNRKGYKAGNLKSGMECDYVKDCEFVA & $3.7 \mathrm{e}-650$ & 27 & 41 \\
10 & VSLWKKFNLJYLFFFVRKVVAPFYTFTLYCVIJPLSVFVPE & $1.6 \mathrm{e}-573$ & 27 & 41 \\
\hline
\end{tabular}




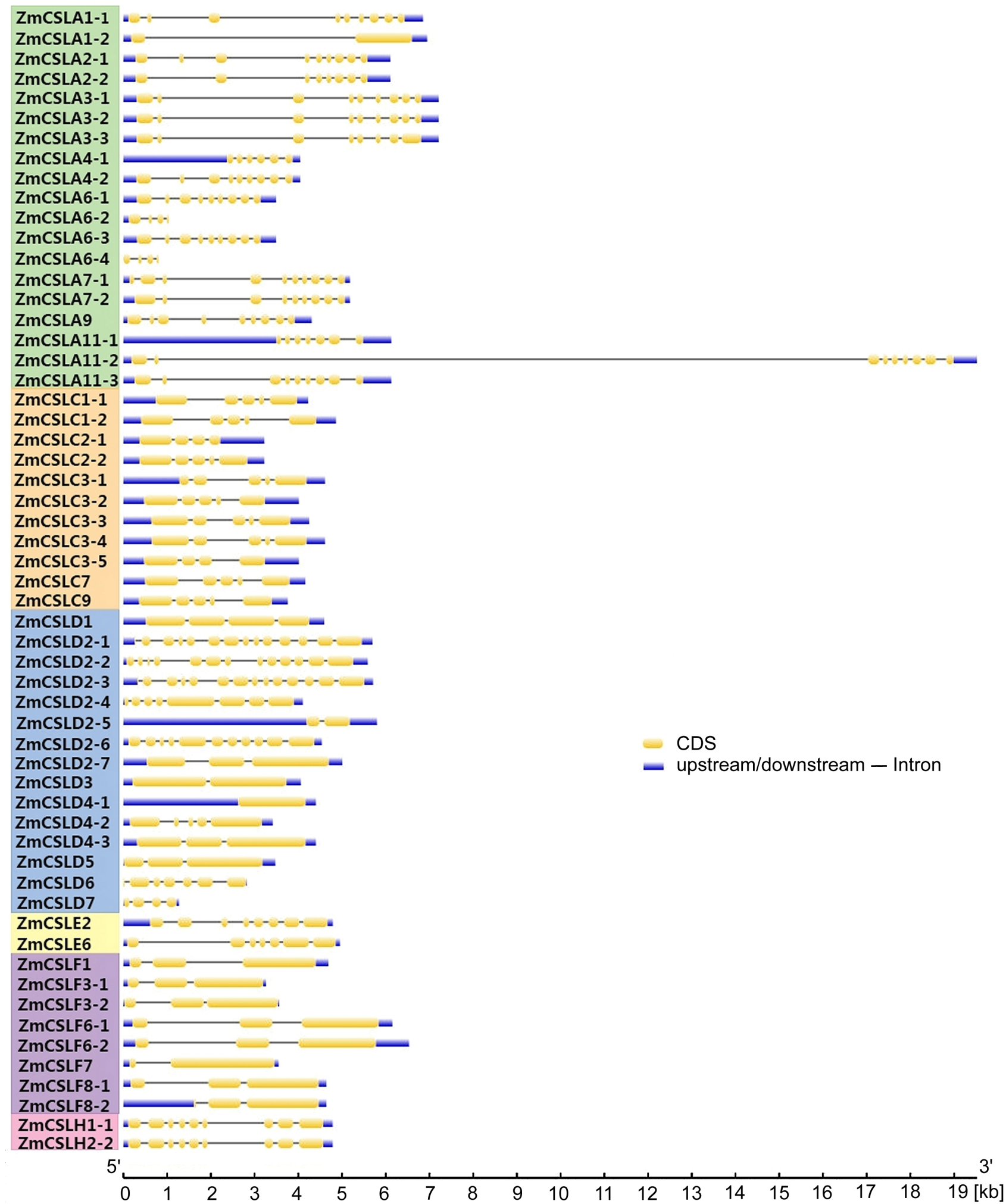

Fig. 2. The exon-intron structure of cellulose synthase-like $(C s l)$ genes in maize. The yellow boxes indicate the exons and the single lines indicate introns. Untranslated regions are displayed by thick purple lines at both ends. Gene models were drawn to the scale as indicated at the bottom. 


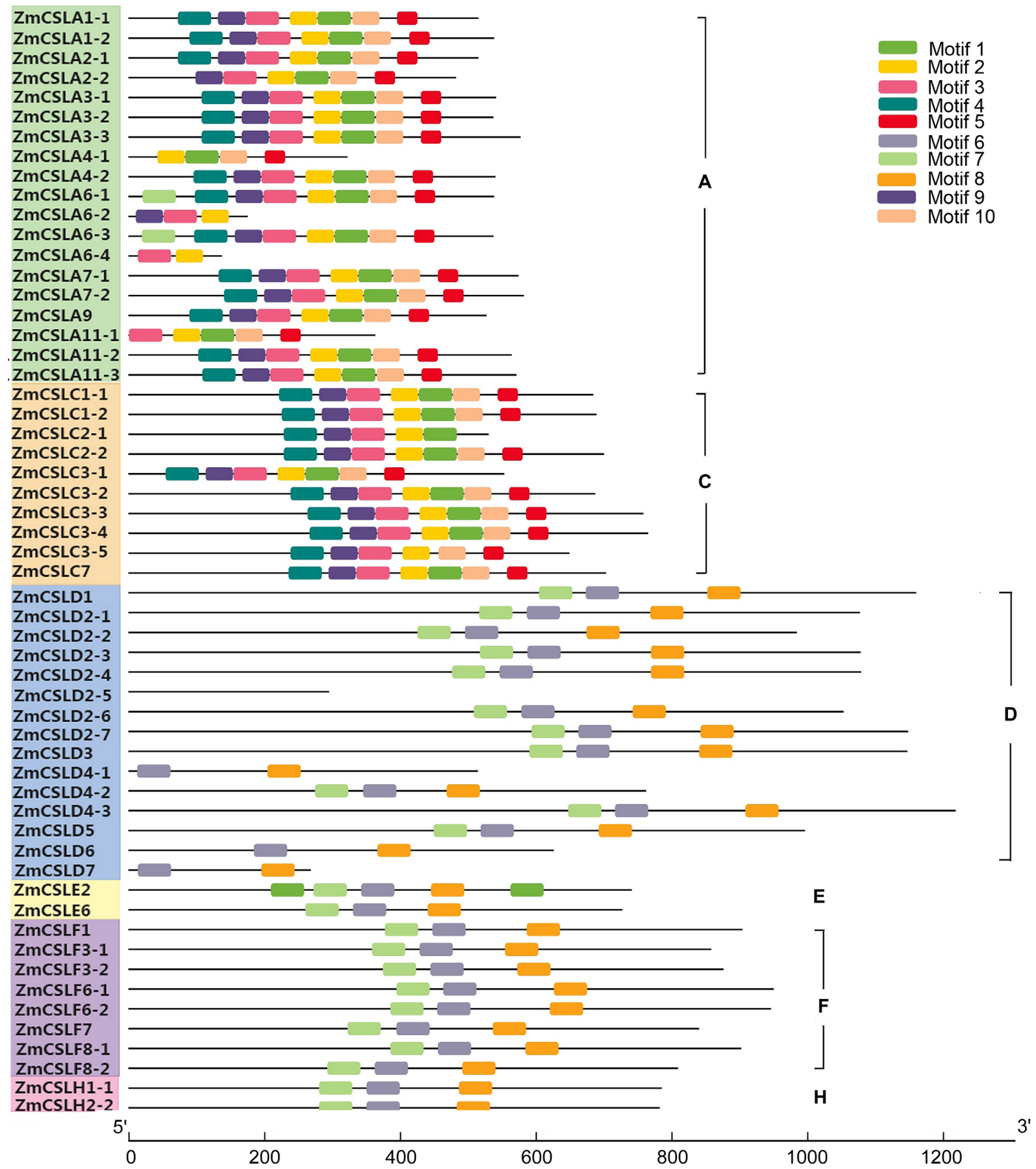

Fig. 3. Conserved motifs of the cellulose synthase-like $(\mathrm{Cs} l)$ gene family in maize. All conserved motifs of the ZmCsl proteins were identified by the $M E M E$ program. Protein sequences are indicated by thick gray lines, and the conserved motifs are represented by different colored boxes. The length (the number of amino acids) of the protein and motif can be estimated using the scale bar at the bottom.

results reveal that the expressions of $\mathrm{ZmCsl}$ genes were responsive to early drought stress.

To illustrate the molecular mechanisms of $\mathrm{ZmCsl}$ proteins, PPI network was constructed using the data from the STRING database (Fig. 7). Four ZmCsl proteins ZmCslD2-4, ZmCslA4-1, ZmCslF6-1, and ZmCslF6-2 


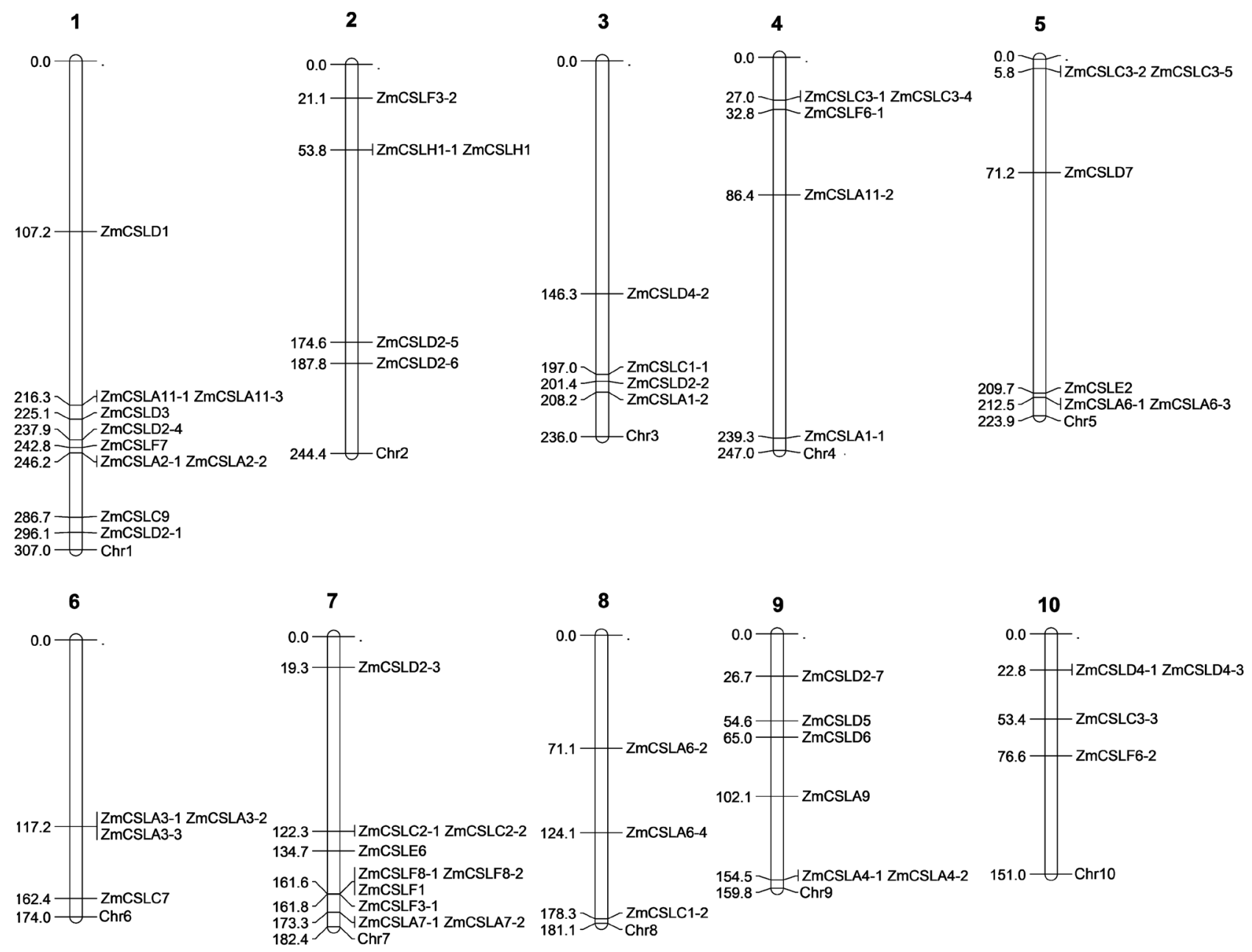

Fig. 4. The chromosomal locations of cellulose synthase-like (Csl) genes in the maize genome. Chromosomes $1-10$ are depicted as bars. The $\mathrm{ZmCsl}$ genes are indicated by black lines.

were included in the network. Furthermore, ZmCslD2-4, GRMZM2G467943 P01, and GRMZM2G025016 P01 were located in the more important positions of the network suggesting those proteins play critical roles in the maintaining the whole protein interactions in the network. However, the function of GRMZM2G467943_P01 and GRMZM2G025016_P01 were unknown. The results in this study were beneficial to identify more important proteins and biological modules that interacted with $\mathrm{ZmCsl}$ proteins. Additionally, some proteins, such as Zm.21995 and GRMZM5G821988_P01 (eukaryotic translation initiation factors), interacted with $\mathrm{ZmCslD2-4}$ as well. The detailed information of the proteins in the PPI network is listed in Table 2 Suppl.

\section{Discussion}

In recent years, a genome-wide analysis of gene family has become an efficient approach to understand gene structure, function, and evolution. The $C s l$ genes are reported to be involved in cellular components and various biological processes in many plant species. However, the comprehensive analysis of $\mathrm{Cs} l$ gene family has been limited to Arabidopsis thaliana, rice, and wheat. In this study, we conducted a comprehensive analysis of the $\mathrm{ZmCsl}$ gene family, including identification of members, phylogenetic relationships, chromosomal organization, and expression profiles in different tissues and under drought stress conditions. The results will assist in understanding the roles of these $\mathrm{ZmCsl}$ genes and their potential molecular mechanisms in response to drought stress.

A total of 56 putative $C s l$ genes were identified in the maize genome, the number of which was distinct from A. thaliana (36) and rice (34), a dicotyledonous and monocotyledonous plant, respectively (Schwerdt et al. 2015, Kaur et al. 2017). Comparison of the maize genome (2 $500 \mathrm{Mb})$ with A. thaliana $(125 \mathrm{Mb})$ and rice (466 $\mathrm{Mb})$ reveals that maize has about 25-times and 5-times larger genome size than $A$. thaliana and rice, respectively (Inititiative 2000, Sasaki et al. 2002, Shukla et al. 2009). However, the number of $C s l$ genes in these species is not proportional with the genome size. The reason may be that the maize genome has undergone significant gene losses 


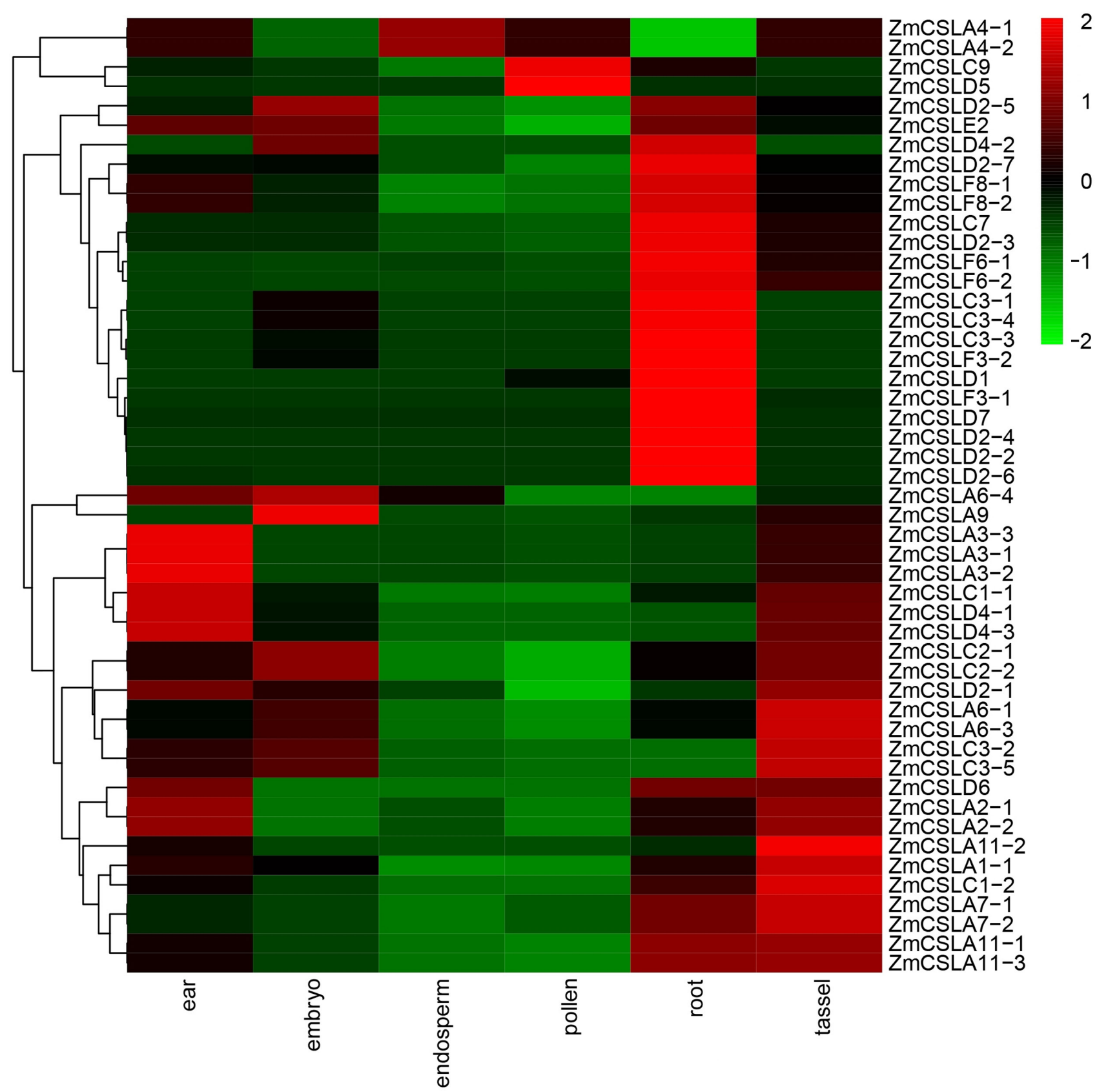

Fig. 5. The expression profiles of maize cellulose synthase-like ( $\mathrm{ZmCs} l$ ) genes across different tissues. The $\mathrm{ZmCsl}$ genes and color scale are shown at the right of the heat map. The genes with fragments per kilobase of exons per million fragments mapped (FPKM) equal to 0 were not used in this heat map.

since the duplication event. The results from Lai et al. (2004) further confirmed this speculation showing that only a small proportion of genes were conserved as duplicate factors in orthologous intervals of maize, when comparing the two homoeologous regions of the maize genome and the single homoeologous regions of the sorghum and rice genomes (Lai et al. 2004, Messing et al. 2004). Besides, phylogenetic analysis divided the members of the $\mathrm{ZmCsl}$ gene family into six distinct groups (CslA, CslC, CslD, $C s l E, C s l F$, and $C s l H)$, which are largely similar to those found in rice whereas $C s l$ genes in A. thaliana are divided into other six groups (CslA, CslB, CslC, CslD, CslE, and $C s l G)$. The results suggest that $C s l$ genes may have a potential functional diversity between dicot and monocot plants (Penning et al. 2009).

The role of $C s l$ genes have been reported mainly in A. thaliana and rice. For example, by destroying CsLD5 expression in A. thaliana, Bernal et al. (2010) found that the growth of stem and root is significantly reduced in CslD5 knockout plants, and the activities of xylan synthase and homogalacturonan synthase are also reduced indicating a possible role of this gene and other $C s l D$ genes in cell wall 


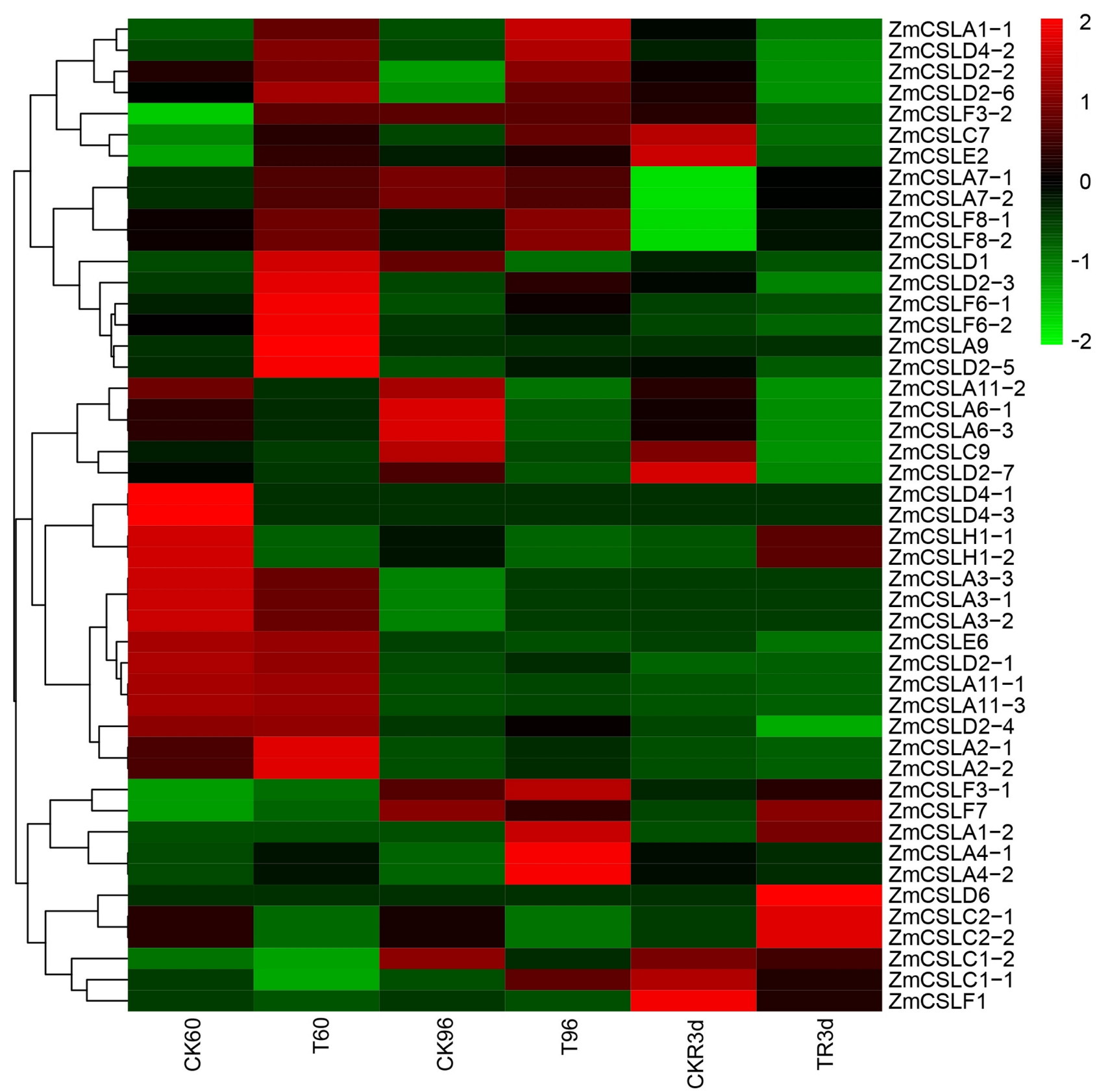

Fig. 6. The expression profiles of maize cellulose synthase-like ( $\mathrm{ZmCsl}$ ) genes in response to drought stress treatment. The color scale is shown at the right of the heat map. A higher expression for each gene is presented in red; otherwise, green was used. T60, T96, and TR3d indicate samples treated with PEG for 60 or $96 \mathrm{~h}$ and then recovered for $3 \mathrm{~d}$. Control seedlings were grown under the same conditions but without PEG treatment (denoted as CK60, CK96h, and CKR3d, respectively).

biosynthesis. Goubet et al. (2010) observed glucomannan deficiency in a CslA mutant of $A$. thaliana indicating that the CslA family codes for glucomannan synthetase. In rice, there are continuous researches on the $C s l$ gene family since the $C s l$ gene family in rice has been identified (Hazen et al. 2002). For example, Wang et al. (2010) found that $C s l$ genes in rice play important roles in the potentially functional complement for cell wall synthesis using transcriptional profiling and co-expression analyses. However, limited researches of $C s l$ genes focused on maize with the exception of $Z m C s l D 1$, which is integral to normal cell division, expansion, leaf development, and controls organ size in maize (Hunter 2010, Li et al. 2018).

In this study, a systematic analysis of $\mathrm{ZmCsl}$ genes was performed including the identification of members, phylogenetic analysis, and gene structure analysis. Furthermore, we explored the expression evidence for all putative $\mathrm{ZmCsl}$ genes in different tissues (ear, embryo, endosperm, pollen, root, and tassel) using the publicly available transcriptome data. The $Z m C s l D$ and $Z m C s l F$ 


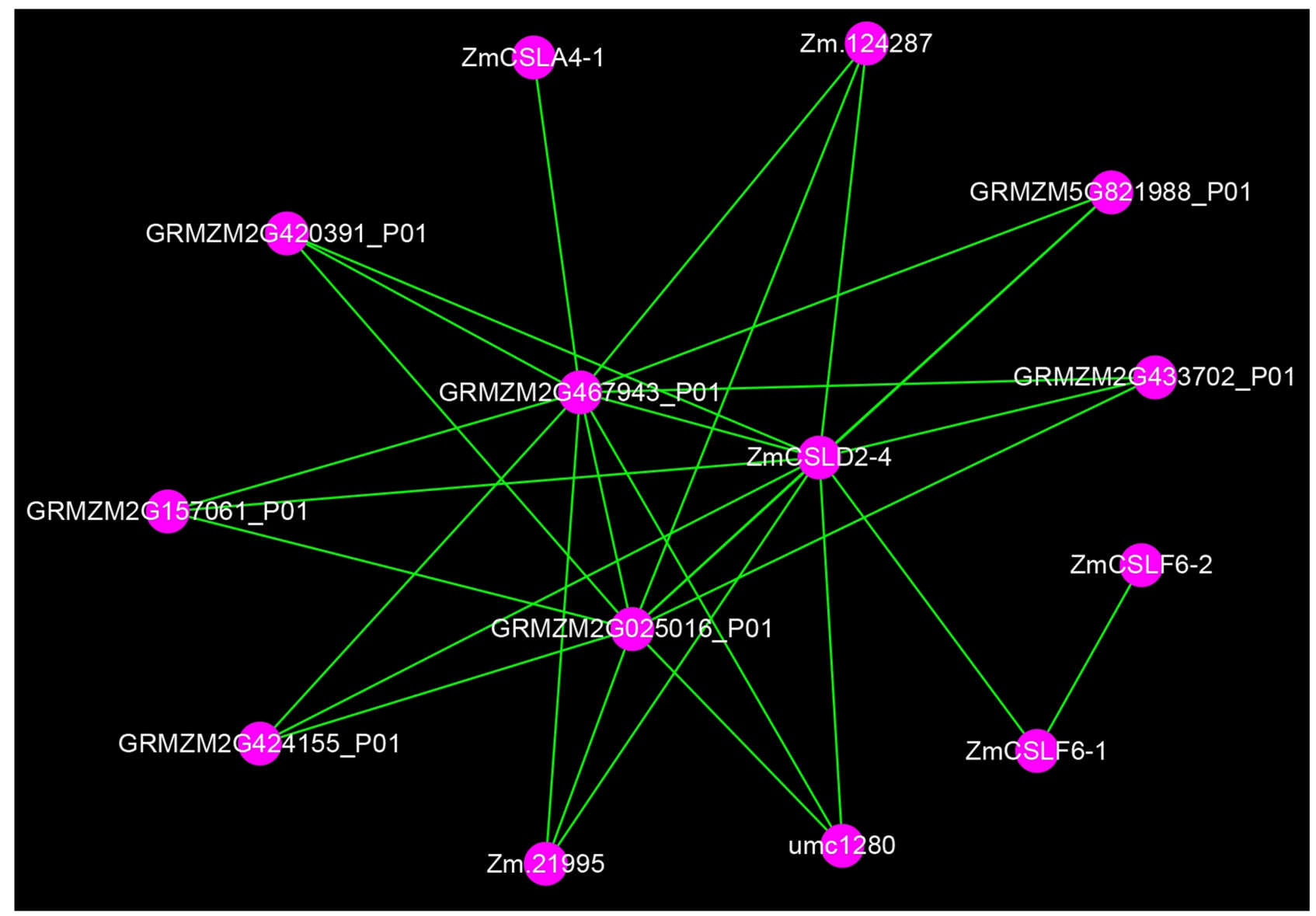

Fig. 7. A protein-protein interactions (PPI) network of maize cellulose synthase-like ( $\mathrm{ZmCsl}$ ). The nodes represent proteins and the edges represent the corresponding PPI. The confidence score was required to be greater than 0.7 .

genes exhibited relatively high expressions in root tissue consistent with a previous research indicating that they could play a role in the development of tplant root (Wang et al. 2001, Hansey et al. 2010). Moreover, Csl genes have been shown to play crucial roles in responses to various biotic and abiotic stresses. In A. thaliana, AtCslD5 is not essential for normal growth and development but play a critical role in osmotic stress tolerance, which may be likely involved in the regulation of reactive oxygen species under stress (Zhu et al. 2010). In another study of A. thaliana, a cellulose synthase gene was reported to enhance drought and osmotic stress tolerance through regulating cellulose synthesis (Chen et al. 2010). In Hordeum vulgare, HvCslD2 represents an important defensive reaction both for nonhost resistance and for quantitative host resistance against nonadapted wheat and host-adapted barley powdery mildew pathogens, respectively, through mediating cell wall changes in the epidermal layer (Douchkov et al. 2016). In the present study, a significant number of $\mathrm{ZmCsl}$ genes was up-regulated after drought treatment for $60 \mathrm{~h}$ suggesting that these $C s l$ genes may play positive roles in response to early drought stress. However, the specific mechanism of action needs to be further demonstrated and experimentally confirmed.

\section{Conclusions}

This study provides a systematic and comprehensive analysis of the $C s l$ gene family in maize. In total, 56 members of the $C s l$ gene family were identified in the maize genome and were classified into six subfamilies: $C s l A, C s l C, C s l D, C s l E, C s l F$, and CslH. The expression profiles of $\mathrm{ZmCsl}$ genes in different tissues were performed to reveal that most $\mathrm{ZmCsl}$ genes had relatively high expressions in root and tassel tissues. Moreover, the expression patterns of $\mathrm{ZmCsl}$ genes under drought and re-watering indicate that the expressions of $\mathrm{ZmCsl}$ genes were mainly responsive to early stage of drought stress. The detailed description of maize $C s l$ genes will be beneficial to understand their structural, functional, and evolutionary features. This information will provide an important foundation for studying the roles of these $\mathrm{ZmCsl}$ genes in response to biotic and abiotic stresses.

The sequence data are available in the NCBI Sequence Read Archive under accession numbers of SRP067440 for various maize tissues and SRR6665368 to SRR6665379 for maize under drought and recovery. 


\section{References}

Bernal, A.J., Jensen, J.K., Harholt, J., Sørensen, S., Moller, I., Blaukopf, C., Bo, J., Lotto, R.D., Pauly, M., Scheller, H.V.: Disruption of ATCSLD5 results in reduced growth, reduced xylan and homogalacturonan synthase activity and altered xylan occurrence in Arabidopsis. - Plant J. 52: 791-802, 2010.

Chen, Z., Hong, X., Zhang, H., Wang, Y., Li, X., Gong, Z.: Disruption of the cellulose synthase gene, AtCesA8/IRX1, enhances drought and osmotic stress tolerance in Arabidopsis. - Plant J. 43: 273-283, 2010.

Cocuron, J. C., Lerouxel, O., Drakakaki, G., Alonso, A.P., Liepman, A.H., Keegstra, K., Raikhel, N., Wilkerson, C.G.: A gene from the cellulose synthase-like $C$ family encodes a beta-1,4 glucan synthase. - Proc. nat. Acad. Sci. USA 104: 8550-8555, 2007.

Djerbi, S., Lindskog, M., Arvestad, L., Sterky, F., Teeri, T.T.: The genome sequence of black cottonwood (Populus trichocarpa) reveals 18 conserved cellulose synthase $(C e s A)$ genes. - Planta 221: 739-746, 2005

Douchkov, D., Lueck, S., Hensel, G., Kumlehn, J., Rajaraman, J., Johrde, A., Doblin, M.S., Beahan, C.T., Kopischke, M., Fuchs, R.: The barley (Hordeum vulgare) cellulose synthaselike D2 gene ( $\mathrm{HvCs} \mathrm{CD} 2)$ mediates penetration resistance to host-adapted and nonhost isolates of the powdery mildew fungus. - New Phytol. 212: 421-433, 2016.

Goubet, F., Barton, C.J., Mortimer, J.C., Yu, X., Zhang, Z., Miles, G. ., Richens, J., Liepman, A.H., Seffen, K., Dupree, P.: Cell wall glucomannan in Arabidopsis is synthesised by CSLA glycosyltransferases, and influences the progression of embryogenesis. - Plant J. 60: 527-538, 2010.

Hansey, C.N., Lorenz, A.J., Leon, N.D.: Cell wall composition and ruminant digestibility of various maize tissues across development. - Bioenergy Res. 3: 28-37, 2010.

Hazen, S.P., Scottcraig, J.S., Walton, J.D.: Cellulose synthaselike genes of rice. - Plant Physiol. 128: 336-340, 2002.

Hunter, C., Iii, T.: The Mu transportation of Zea mays and their use in determining gene function: cellulose synthase-like D genes in plant and cell development. - Dissertations \& Theses, Gradworks, Floroda 2010.

Inititiative, T.A.G.: Analysis of the genome sequence of Arabidopsis thaliana. - Nature 408: 796-815, 2000.

Kaur, S., Dhugga, K.S., Beech, R., Singh, J.: Genome-wide analysis of the cellulose synthase-like $(C s l)$ gene family in bread wheat (Triticum aestivum L.). - BMC Plant Biol. 17: 193, 2017.

Kaur, S., Dhugga, K.S., Gill, K., Singh, J.: Novel structural and functional motifs in cellulose synthase (CesA) genes of bread wheat (Triticum aestivum L.). - Plos ONE 11: e0147046, 2016.

Lai, J., Ma, J., Swigonová, Z., Ramakrishna, W., Linton, E., Llaca, V., Tanyolac, B., Park, Y.J., Jeong O.Y., Bennetzen, J.L.: Gene loss and movement in the maize genome. - Genome Res. 14: 1924-1931, 2004.

Li, W., Yang, Z., Yao, J., Li, J., Song, W., Yang, X.: Cellulose synthase-like D1 controls organ size in maize. - BMC Plant Biol. 18: 239, 2018.

Liepman, A.H., Wilkerson, C.G., Keegstra, K., Kende, H.J.: Expression of cellulose synthase-like $(C s l)$ genes in insect cells reveals that CslA family members encode mannan synthases. - Proc. nat. Acad. Sci. USA 102: 2221-2226, 2005.

Messing, J., Bharti, A K., Karlowski, W.M., Gundlach, H., Kim, H.R., Yu, Y., Wei, F., Fuks, G., Soderlund, C.A., Mayer, K.F.: Sequence composition and genome organization of maize. Proc. nat. Acad. Sci. USA 101: 14349-14354, 2004.

Penning, B.W., Hunter, C.T., Reuben, T., Eveland, A.L., Dugard, C.K., Olek, A.T., Wilfred, V., Koch, K.E., Mccarty, D. R.,
Davis, M.F.: Genetic resources for maize cell wall biology. Plant Physiol. 151: 1703-1728, 2009.

Roberts, A.W., Bushoven, J.T.: The cellulose synthase (CESA) gene superfamily of the moss Physcomitrella patens. - Plant mol. Biol. 63: 207-219, 2007.

Sasaki, T., Matsumoto, T., Yamamoto, K., Sakata, K., Baba, T., Katayose, Y., Wu, J., Niimura, Y., Cheng Z., Nagamura, Y.: The genome sequence and structure of rice chromosome 1. Nature 420: 312-316, 2002.

Schreiber, M., Wright, F., Mackenzie, K., Hedley, P.E., Schwerdt, J. G., Little, A, Burton, R.A., Fincher, G.B., Marshall, D., Waugh, R., Halpin, C.: The barley genome sequence assembly reveals three additional members of the CslF $(1,3 ; 1,4)-\beta$-glucan synthase gene family. - Plos ONE 9 : e90888, 2014

Schwerdt, J.G , Mackenzie, K, Wright, F., Oehme, D., Wagner, D., Harvey, A.J., Shirley, N.J., Burton, R.A., Schreiber, M., Halpin, C., Zimmer, J., Marshall, D.F., Waugh,R., Fincher, G.: Evolutionary dynamics of the cellulose synthase gene superfamily in grasses. - Plant Physiol. 168: 968-983, 2015.

Shukla, V.K., Doyon, Y., Miller, J.C., Dekelver, R.C., Moehle, E.A., Worden, S.E., Mitchell, J.C., Arnold, N.L., Gopalan, S., Meng, X.: Precise genome modification in the crop species Zea mays using zinc-finger nucleases. - Nature 459: 437-441, 2009.

Suzuki, S., Li, L., Sun, Y.H., Chiang, V.L.: The cellulose synthase gene superfamily and biochemical functions of xylem-specific cellulose synthase-like genes in Populus trichocarpa. - Plant Physiol. 142: 1233-1245, 2006.

Tamura, K., Peterson, D., Peterson, N., Stecher, G., Nei, M., Kumar, S.: MEGA5: molecular evolutionary genetics analysis using maximum likelihood, evolutionary distance, and maximum parsimony methods. - Mol. Biol. Evol. 28: 2731, 2011.

Vega-Sánchez, M.E., Verhertbruggen, Y., Christensen, U., Chen, X., Sharma, V., Varanasi, P., Jobling, S.A., Talbot, M., White, R.G., Joo, M.: Loss of cellulose synthase-like F6 function affects mixed-linkage glucan deposition, cell wall mechanical properties, and defense responses in vegetative tissues of rice. - Plant Physiol. 159: 56-69, 2012.

Verhertbruggen, Y., Yin, L., Oikawa, A., Scheller, H.V.: Mannan synthase activity in the CSLD family. - Plant Signal. Behav. 6: 1620-1623, 2011.

Voorrips, R.E., MapChart: software for the graphical presentation of linkage maps and QTLs. - J. Heredity 93: 77-78, 2002.

Wang, B., Tseng, E., Regulski, M., Clark, T.A., Hon, T., Jiao, Y., Lu, Z., Olson, A., Stein, J.C., Ware, D.: Unveiling the complexity of the maize transcriptome by single-molecule long-read sequencing. - Nat. Commun. 7: 11708, 2016.

Wang, L., Guo, K., Li, Y., Tu, Y., Hu, H., Wang, B., Cui, X., Peng, L.: Expression profiling and integrative analysis of the CESA/ CSL superfamily in rice. - BMC Plant Biol. 10: 282, 2010.

Wang, X., Cnops, G., Vanderhaeghen, R., Block, S.D.: AtCSLD3, a cellulose synthase-like gene important for root hair growth in Arabidopsis. - Plant Physiol. 126: 575-586, 2001.

Wright, S.I., Bi, I.V., Schroeder, S.G., Yamasaki, M., Doebley, J.F., Mcmullen, M.D., Gaut, B.S.: The effects of artificial selection on the maize genome. - Science 308: 1310-1314, 2005.

Wu, Z., Cheng, J., Cui, J., Xu, X., Liang, G., Luo, X., Chen, X., Tang, X., Hu, K., Cheng, Q.: Genome-wide identification and expression profile of Dof transcription factor gene family in Pepper (Capsicum annuum L.). - Front. Plant Sci. 7: 574, 2016.

Yin, Y., Huang, J., Xu, Y.: The cellulose synthase superfamily in fully sequenced plants and algae. - BMC Plant Biol. 9: 99, 
2009.

Yin, Y., Johns, M.A., Cao, H., Rupani, M.: A survey of plant and algal genomes and transcriptomes reveals new insights into the evolution and function of the cellulose synthase superfamily. - BMC Genomics 15: 260, 2014.

Zhou, C., Yin, Y., Dam, P., Xu, Y.: Identification of novel proteins involved in plant cell-wall synthesis based on protein-protein interaction data. - J. Proteome Res. 9: 5025-5037, 2010.
Zhu, J., Lee, B.H., Dellinger, M., Cui, X., Zhang, C., Wu, S., Nothnagel, E.A., Zhu, J.K.A.: Cellulose synthase-like protein is required for osmotic stress tolerance in Arabidopsis. - Plant J. 63: 128-140, 2010.

Zhu, G., Wu, A., Xu, X., Xiao, P., Lu, L., Liu, J., Cao, Y., Chen, L., Wu, J., Zhao, X.: PPIM: A protein-protein interaction database for maize. - Plant Physiol. 170: 618-626, 2016. 